\title{
Endosalpingiosis de cicatriz operatoria de Pfannenstiel. Reporte de caso
}

\author{
María del Pilar Arciénega Yáñez*, Daniel González González
}

\section{Resumen}

Introducción: la endosalpingiosis es una enfermedad benigna caracterizada por el crecimiento ectópico del epitelio de la trompa de Falopio, siendo su presencia en cicatrices operatorias excepcional, habiéndose reportado cuatro casos en la literatura internacional.

Caso clínico: se presenta el caso de una paciente de 40 años que consultó por dolor sobre incisión de Pfannenstiel y tumoración a dicho nivel. La misma se resecó y el estudio anatomopatológico informó endosalpingiosis.

Discusión: el diagnóstico de endosalpingiosis cutánea es posoperatorio y realizado luego del estudio de la pieza de resección. Con su extirpación completa, se resuelve definitivamente la sintomatología y en los pocos casos publicados en la literatura no se ha reportado recidiva.

Palabras clave: Endosalpingiosis Procedimientos quirúrgicos ginecológicos Cicatriz

Enfermedades de la piel

Key words: Endosalpingiosis

Gynecologic surgical procedures

Cicatrix

Skin diseases

\section{Introducción}

El término endosalpingiosis fue empleado por primera vez por Sampson ${ }^{(1)}$ en 1930, quien denominó bajo este término cualquier crecimiento ectópico e invasión del epitelio de trompas de Falopio en mujeres que se habían sometido a salpingectomía o esterilización tubaria. Histológicamente se define por la presencia de estructuras quísticas cubiertas por epitelio ciliado similar al de las trompas de Falopio ${ }^{(2)}$.

La endosalpingiosis, junto a la endometriosis y endocervicosis, constituyen la tríada de los trastornos no neoplásicos del sistema de Müller, que si bien se pueden presentar de forma aislada, en oportunidades se asocian $^{(3)}$. El diagnóstico de endosalpingiosis es histológico y realizado luego de la resección quirúrgica.

El objetivo es comunicar un caso de esta infrecuente entidad clínica de la cual existen publicados en la literatura internacional solo cuatro casos ${ }^{(3-6)}$.

\section{Caso clínico}

Paciente de sexo femenino, 40 años, con antecedentes de apendectomía a los 17 años y dos cesáreas, la última realizada seis años previos a la consulta, donde además se realizó ligadura tubárica.

Consultó por una historia de un año de evolución dada por dolor a nivel de la comisura derecha de la incisión de Pfannenstiel, permanente, de escasa intensidad, sin cambios en relación al ciclo menstrual y que posteriormente agregó una pequeña tumoración a dicho nivel. $\mathrm{Al}$ examen abdominal se constató una tumefacción bien delimitada, dolorosa, de 1 por $1 \mathrm{~cm}$ que no desaparecía con

\footnotetext{
* Residente de Cirugía General.

† Profesor titular de Clínica Quirúrgica.

Clínica Quirúrgica 3. Facultad de Medicina, Universidad de la República, Hospital Maciel. Montevideo, Uruguay.

Los autores declaran no tener conflicto de intereses.

Se cuenta con el consentimiento informado del paciente.

Correspondencia: Pilar Arciénega. Correo electrónico: pily_arya@hotmail.com

Recibido: 20/5/19

Aprobado: 15/7/19
} 


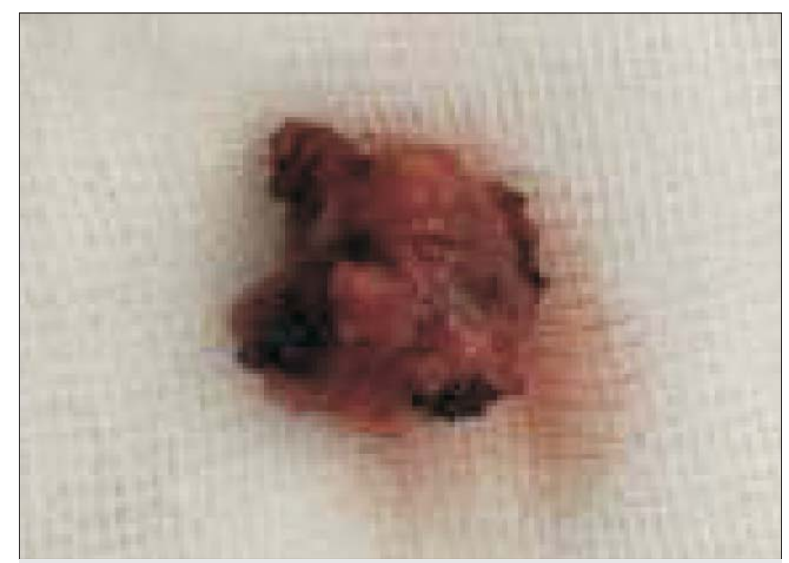

Figura 1. Pieza de resección.

la contracción muscular, manteniendo sus características.

Se realizó ecografía de partes blandas que informó: sobre comisura derecha de cicatriz operatoria de Pfannenstiel se constata imagen nodular sólida, ovoidea, de contornos regulares y bien definidos, de ecogenicidad heterogénea, predominantemente hipoecogénica de 14 por 7 por 10 milímetros, siendo compatible con un granuloma.

Se realizó cirugía de coordinación, resecándose la tumoración en forma completa con anestesia local a través de una incisión centrada en ella. Al corte se comprobó que se trataba de una tumefacción quística (figuras 1 y 2).

Presentó buena evolución posoperatoria, sin complicaciones a nivel de la herida operatoria, y remisión completa de la sintomatología.

El estudio anatomopatológico de la pieza informó: estructuras quísticas revestidas por epitelio columnar simple, con células ciliadas y presencia de células claras. En su luz se observan detritus celulares, hemosiderina y cristales de colesterol. Se observan sectores de fibrosis y leve infiltrado inflamatorio de tipo mixto. Elementos histopatológicos compatibles con una endosalpingiosis (figura 3). Ausencia de elementos de malignidad.

\section{Discusión}

En la literatura internacional se han reportado cuatro casos de endosalpingiosis cutánea ${ }^{(3-7)}$.

En cuatro de los casos existió el antecedente de una cirugía de la esfera ginecológica, dos cesáreas, una salpingectomía y miomectomía. De los restantes, en uno se plantea que hubo una interrupción del embarazo pero no se explicita la vía ${ }^{(6)}$, y finalmente, en otro, no hubo antecedente quirúrgico $^{(7)}$.

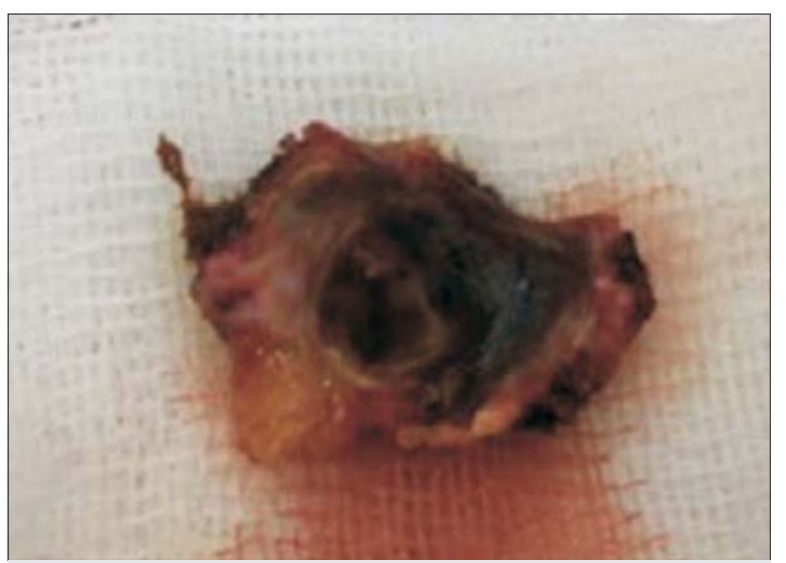

Figura 2. Pieza de resección abierta.

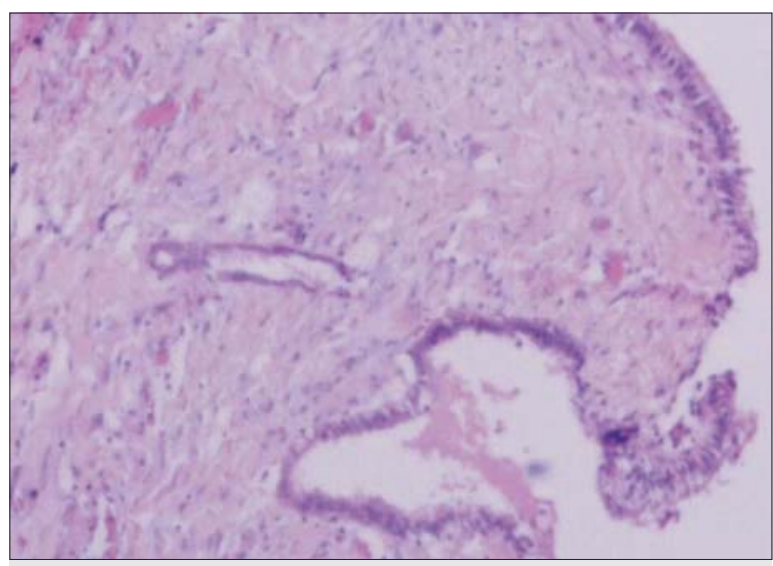

Figura 3. Microscopía de la pieza: epitelio columnar ciliado. Hematoxilina-eosina. 60x.

El rango de edad en los cuales se presentó fue de 30 a 53 años, estando nuestro caso comprendido dentro del rango.

El dolor fue el síntoma principal, habiéndose presentado en el 50\% de los casos, alcanzando duraciones de varios años en la mayoría de ellos, a excepción de uno que fue intenso y que se instaló cinco días previos a la consulta $^{(5)}$.

Como hecho destacable cabe señalar que la tumoración en ninguno de los casos reportados previamente asentaron sobre una cicatriz de Pfannenstiel, tres de ellos en la región umbilical y en otro a nivel inguinal, donde se había emplazado un tubo de drenaje, por lo que constituiría el primer caso en dicha localización. Si bien la etiopatogenia puede ser debatida, y teniendo en cuenta las dos teorías aceptadas ${ }^{(7,8)}$, parecería ser que nuestro caso se ajusta a la del transporte de las células desde la trompa y posterior implante en la cicatriz operatoria, ya 
sea por medio del instrumental quirúrgico utilizado o las propias manos del cirujano.

Habitualmente el tamaño del nódulo es pequeño, y a excepción de uno que alcanzó los $4 \mathrm{~cm}$, los restantes fueron menores a $1 \mathrm{~cm}$.

La presencia de un epitelio tubular con células columnares ciliadas, columnares no ciliadas secretoras y las denominadas intercalarias o peg en la pieza de resección quirúrgica, confirman el diagnóstico.

El tratamiento es quirúrgico y es factible realizarlo con anestesia local, siendo condición indispensable su resección completa. Bajo estas circunstancias, no se han reportado recurrencias.

\section{Summary}

Introducion: endosalpingiosis is a benign disease characterized by the ectopic growth of the epithelium in the Fallopian tube, being it exceptional in surgical scars, four cases having been reported by international literature.

Clinical case: the study presents the clinical case of a 40 year old female patient who consulted for pain in the Pfannenstiel incision and a tumour on the site. After resection, pathological study revealed endosalpingiosis.

Discussion: the diagnosis of cutaneous endosalpingiosis occurs after surgery and upon the analysis of the resected piece. Complete removal results in the final solution of symptoms and no relapse has been reported in cases published in literature.

\section{Resumo}

Introdução: a endossalpingiose é uma condição benigna que se caracteriza pelo crescimento ectópico do epitélio da trompa de Falópio; sua presença em cicatrizes operatórias é excepcional sendo que somente quatro casos foram encontrados na literatura internacional.
Caso clínico: apresenta-se o caso clínico de uma paciente de 40 anos de sexo feminino que consultou por dor e tumoração na incisão de Pfannenstiel. O laudo de anatomia patológico da tumoração ressecada foi endossalpingiose.

Discussão: o diagnóstico de endossalpingiose cutânea é pós-operatório e é feito pelo exame da peça de ressecção. Sua extirpação completa soluciona definitivamente a sintomatologia; os poucos casos publicados na literatura não informam recidivas.

\section{Bibliografía}

1. Sampson JA. Postsalpingectomy endometriosis (endosalpingiosis). Am J Obstet Gynecol 1930; 20(4):443-80

2. Nixon KE, Kenneth Schoolmeester J, Bakkum-Gamez JN. Florid cystic endosalpingiosis with uterine preservation and successful assisted reproductive therapy. Gynecol Oncol Rep 2018; 25:8-10.

3. Stojanovic M, Brasanac D, Stojicic M. Cutaneous inguinal scar endosalpingiosis and endometriosis: case report with review of literature. Am J Dermatopathol 2013; 35(2):254-60.

4. Doé N, Landry M, Cadotte M, Schürch W. Cutaneous endosalpingiosis. Arch Dermatol 1980; 116(8):909-12.

5. Redondo $\mathbf{P}$, Idoate $\mathbf{M}$, Corella $\mathbf{C}$. Cutaneous umbilical endosalpingiosis with severe abdominal pain. J Eur Acad Dermatol Venereol 2001; 15(2):179-80.

6. Perera GK, Watson KM, Salisbury J, Du Vivier AW. Two cases of cutaneous umbilical endosalpingiosis. Br J Dermatol 2004; 151(4):924-5.

7. Papavramidis TS, Sapalidis K, Michalopoulos N, Karayannopoulou G, Cheva A, Papavramidis ST. Umbilical endosalpingiosis: a case report. J Med Case Rep 2010; 4:287.

8. Amir RAR, Taheini KM, Sheikh SS. Mullerianosis of the urinary bladder: a case report. Case Rep Oncol 2018; 11(1):206-11.

María del Pilar Arciénega Yáñez, https://orcid.org/0000-0002-1981-3402.

Daniel González González, https://orcid.org/0000-0003-3916-9201. 